\title{
Evaluation of sensitivity of 10 diagnostic assays for Chlamydia trachomatis by use of a simple laboratory procedure
}

\author{
B J Thomas, E J MacLeod, D Taylor-Robinson
}

\begin{abstract}
Aims-To determine the sensitivity of commercially available diagnostic assays for Chlamydia trachomatis using a simple method.

Methods-Nine commercial assays and an "in-house" polymerase chain reaction (PCR) were evaluated using serial dilutions of a laboratory grown $H$ serovarfour of them using a laboratory grown $E$ serovar. Seven of the assays were further tested using dilutions of several cervical samples known to contain chlamydiae.

Results-The most sensitive assays were the MicroTrak direct fluorescent antibody (DFA) test (Syva) and the PCR which detected $C$ trachomatis at a $10^{-8}$ dilution of the $H$ serovar, while the two least sensitive, Clearview (Unipath) and TestPack (Abbott), were positive only at $10^{-4}$ and $10^{-3}$ dilutions, respectively. A range of enzyme immunoassays (EIAs) and a nucleic acid hybridisation test were of intermediate sensitivity. The results with serovar $E$ were consistent with these. When clinical samples were examined, the DFA test detected $C$ trachomatis in dilutions at least 10 -fold greater than any other assay.

Conclusions-The range of sensitivity of diagnostic assays determined by the laboratory dilution procedure is very wide. Sensitivity assessed in this way, however, reflects the ability of the assays to detect $C$ trachomatis in large scale clinical trials. The dilution procedure, which is simple to undertake, could therefore be applied by any laboratory before a new diagnostic method is considered for routine use.
\end{abstract}

(F Clin Pathol 1993;46:912-914)

persion of this paper was published in published here is the correct one, and the previous version should be ignored.

Division of Sexually

Transmitted Diseases, Clinical Research Centre, Harrow, Middlesex, and The Jefieriss Wing, St Mary's Hospital, Paddington, London W2 INY

B J Thoma

E J MacLeod

D Taylor-Robinson

Correspondence to:

Dr B J Thomas

Accepted for publication

21 October 1992

Many factors influence the decision to adopt a particular diagnostic test for Chlamydia trachomatis. These include the number of samples to be processed, the cost of each test, and the range of other similar assays in use in the laboratory. Above all these considerations, however, the sensitivity and specificity of any new assay must be satisfactory. In view of this, it is unfortunate that a clinician often has to rely for this vital information on results published by other workers who have compared the new test with a diagnostic method different from that used in the clinician's that large scale evaluations are time consuming and expensive and cannot be undertaken by every laboratory or clinic for each new assay that becomes available commercially.

Although the specificity of diagnostic tests for $C$ trachomatis is clearly important, for most of them the question of sensitivity has been, and is, the greater issue. In view of this we applied a rapid and inexpensive method that we have used before ${ }^{1}$ to assess the relative sensitivities of such tests. The ability of the tests to detect elementary bodies in increasing dilutions of two laboratory stock strains and of clinical samples known to contain elementary bodies was determined. The latter samples were included to take into account soluble lipopolysaccharide (LPS) or free chlamydial ribosomal RNA which might occur in infected patients, but not in cultures of laboratory stock strains. The method allows the sensitivities of two or more assays to be compared directly using identical material, a situation which is impossible to achieve in large scale clinical trials.

\section{Methods}

C TRACHOMATIS SEROVARS

Serovar H (UW4) and serovar E (DK20) of $C$ trachomatis were cultured in cycloheximide treated McCoy cell monolayers, ${ }^{2}$ resuspended in $0.2 \mathrm{M}$ sucrose phosphate buffer (2SP), containing $10 \%$ fetal calf serum, and stored in aliquots in liquid nitrogen.

\section{CLINICAL SAMPLES}

Cervical swab samples were obtained from women attending the genitourinary medicine clinic at St Mary's Hospital, Paddington. Swabs for some assays (IDEIA; PACE 2) were placed directly into transport tubes provided for the assay, and those for others were replaced in dry tubes and transported to the laboratory within six hours. practice. Such reliance derives from the fact
DIAGNOSTIC KITS TESTED

Seven commercially available EIAs, a DNA probe (PACE 2), and a direct fluorescent antibody (DFA) test were evaluated. These diagnostic kits comprised: (1) TestPack (Abbott Laboratories); (2) Clearview (Unipath); (3) Chlamydiazyme (Abbott Laboratories); (4) PACE 2 (Lab Impex); (5) Wellcozyme (Wellcome Laboratories); (6) IDEIA (NovoNordisk); (7) Celisa (Cellabs); (8) MicroTrak (MT) EIA (Syva); and (9) MicroTrak (MT) DFA (Syva). An assay for $C$ trachomatis, involving use of the polymerase 
Table 1 Greatest dilutions of serovar $H$ found positive by indicated diagnostic tests

\begin{tabular}{ll}
\hline Assay & $\begin{array}{l}\text { Greatest dilution of } \\
\text { serovar H positive }\end{array}$ \\
\hline MicroTrak (MT) DFA & $10^{-8}$ \\
PCR & $10^{-8}$ \\
MicroTrak (MT) EIA & $10^{-6}$ \\
Celisa (CE) & $10^{-6}$ \\
IDEIA (ID) & $10^{-5}$ \\
Wellcozyme (WE) & $10^{-5}$ \\
PACE 2 & $10^{-5}$ \\
Chlamydiazyme & $10^{-4}$ \\
Clearview (CV) & $10^{-4}$ \\
TestPack & $10^{-3}$ \\
\hline
\end{tabular}

chain reaction (PCR), which had been developed at the Clinical Research Centre (CRC) before, ${ }^{3}$ was also included.

\section{TEST PROCEDURE}

Use of laboratory grown serovars $H$ and $E$

For assay numbers $3-7$, a $0 \cdot 1 \mathrm{ml}$ aliquot of serovar $\mathrm{H}$ was added to the volume of extraction buffer recommended for each assay. This was considered to be the initial, concentrated sample, from which further 10-fold dilutions $\left(10^{-1}\right.$ to $\left.10^{-8}\right)$ were made in $0.9 \mathrm{ml}$ volumes of extraction buffer. The initial samples and all the dilutions were tested in the assays, following the standard procedures recommended for clinical samples by the manufacturers. After an aliquot of each dilution had been removed for testing, the remainder was centrifuged at $6500 \mathrm{rpm}$ for 10 minutes in a Microfuge (MicroCentaur; MSE). The deposit was transferred to a MicroTrak slide, dried, fixed and then stained with the MicroTrak DFA reagent. The highest dilutions which were found to be positive by each assay and by MicroTrak DFA staining were recorded.

In assays 1 and 2 the whole volume of the initial sample $(1 \mathrm{ml})$ and dilutions in extraction buffer were required for the test, so that none was available for centrifugation; in assay 8 chlamydial elementary bodies were disrupted to release ribosomal RNA, so none would remain intact in the deposit for DFA staining. In these three assays, therefore, double volumes of the initial sample and of all the dilutions were made, one half being used in each assay and the centrifuged deposit from

Table 2 Greatest dilutions * of clinical samples positive by indicated diagnostic tests

\begin{tabular}{|c|c|c|c|c|c|c|}
\hline $\begin{array}{l}M T \\
D F A\end{array}$ & $\begin{array}{l}M T \\
E L A\end{array}$ & $C E$ & $I D$ & $W E$ & $P A C E 2$ & $C V$ \\
\hline $\begin{array}{l}-3 \\
-2 \\
-1 \\
-1 \\
-5 \\
-3 \\
-5 \\
-4 \\
-4 \\
-3 \\
-3 \\
-2 \\
-5 \\
-3 \\
-5 \\
-1 \\
-5\end{array}$ & $\begin{array}{c}-2 \\
-1 \\
U \\
N \\
-4 \\
-2\end{array}$ & $\begin{array}{c}-1 \\
\mathrm{U} \\
\mathrm{N} \\
-4\end{array}$ & $\begin{array}{l}-3 \\
-3 \\
-3\end{array}$ & $\begin{array}{c}N \\
-1 \\
-1\end{array}$ & $\begin{array}{c}-2 \\
\mathbf{N} \\
\mathbf{N} \\
\mathbf{N} \\
-4 \\
-1 \\
-3 \\
-2 \\
-3\end{array}$ & $\underset{-3}{\mathbf{N}}$ \\
\hline
\end{tabular}

${ }^{\star} \log _{10} ; \mathrm{U}=$ undiluted; $\mathrm{N}=$ negative. the other half being stained by the DFA method. Assays 2, 4, 5, 6, 8, and 9 were tested up to four times using different aliquots of the same stock chlamydial pool.

Serovar E was used to test assays $4,7,8$, and 9 in the same manner.

\section{Use of clinical samples}

Swabs for the IDEIA and PACE 2 assays arrived at the laboratory in the respective transport medium. The recommended volume of transport extraction buffer was added to the dry swabs for the Syva EIA, Celisa, Wellcozyme and Clearview tests and the swabs were vortexed in the medium, in each case according to the manufacturer's instructions. The resulting suspension was regarded as the original, undiluted sample and 10-fold dilutions $\left(10^{-1}\right.$ to $\left.10^{-4}\right)$ of it were made in $0.9 \mathrm{ml}$ volumes of extraction buffer. These dilutions, and the original sample, were then tested in all the assays, according to the manufacturer's instructions. As before, the remainder of each sample, or dilution, was centrifuged and the deposit stained by the DFA method. The highest dilution found to contain elementary bodies was recorded.

\section{Results}

\section{DETECTION OF C TRACHOMATIS SEROVARS}

H AND E

The highest dilution of serovar $\mathrm{H}$ found to provide a positive result in each of the nine commercial assays and in the Clinical Research Centre PCR is shown in table 1. Repeated testing of six of the assays provided consistent results indicating the reproducibility of the system. The MT DFA test and the Clinical Research Centre PCR were the most sensitive, $C$ trachomatis being detected at a dilution of $10^{-8}$ of the stock pool. The PACE 2 DNA probe and most of the EIAs were of intermediate sensitivity, elementary bodies being detected at dilutions of $10^{-5}$ to $10^{-6}$. Chlamydiazyme and the two rapid "desk top" assays (Clearview and TestPack) were at the bottom of the range, being positive at only the $10^{-4}$ or $10^{-3}$ dilutions. For all the assays, examination by the MT DFA test of the deposit from the centrifuged $10^{-8}$ dilution remaining after an aliquot had been removed for testing, or examination of the duplicate sample, showed that $\leqslant 10$ elementary bodies were present.

The results with serovar $\mathrm{E}$, which was used to test assays $4,7,8$, and 9 , were consistent with those obtained with serovar $\mathrm{H}$.

\section{DETECTION OF C TRACHOMATIS IN CLINICAL} SAMPLES

The results of testing dilutions of chlamydia positive clinical samples by seven assays are shown in table 2. Use of the MT DFA test resulted in $C$ trachomatis being detected at at least one 10-fold dilution greater than by any other assay. Of three samples which contained small numbers of elementary bodies, detectable only at a $10^{-1}$ dilution by the MT DFA, two were not positive by any of the 
Table 3 Relative sensitivities of diagnostic methods in laboratory tests and clinical trials

\begin{tabular}{lll}
\hline Assay & $\begin{array}{l}\text { Dilution of } \\
\text { serovar } H \text { at } \\
\text { which positive }\end{array}$ & $\begin{array}{l}\text { Sensitivity in } \\
\text { clinical trial }\end{array}$ \\
\hline MT DFA & $10^{-8}$ & Up to $100 \%^{4}$ \\
PCR & $10^{-8}$ & Up to $100 \%^{3}$ \\
MT EIA & $10^{-6}$ & $92 \%^{\star}$ \\
IDEIA & $10^{-5}$ & $74 \%^{6}$ \\
Chlamydiazyme & $10^{-4}$ & $62 \%^{1}$ \\
\hline *Unpublished data; B J Thomas.
\end{tabular}

^Unpublished data; B J Thomas

other assays tested (MT EIA, Celisa, PACE 2 , or Clearview) and the third was positive only by the MT EIA and the Celisa when the sample was undiluted.

\section{Discussion}

The results of testing dilutions of the laboratory grown serovars of $C$ trachomatis show the enormous variation in the ability of diagnostic tests to detect elementary bodies. Indeed, with the most sensitive, at the top of the range, fewer than 10 elementary bodies were detected at a $10^{-8}$ dilution, while with tests of less sensitivity, elementary bodies could be detected only at dilutions $10^{2}-10^{3}$-fold less or even $10^{4}-10^{5}$-fold less. In our hands, this inability to detect small numbers of elementary bodies is reflected directly in the poor sensitivity values recorded for certain assays in large scale clinical trials (table 3). In all cases the shortfall in sensitivity can be accounted for by the fact that about $30 \%$ of all clinical samples contain $\leqslant 10$ elementary bodies as judged by examining a smear stained by MT DFA. ${ }^{5}$ Increasing the numbers of elementary bodies available for testing increases the sensitivity of the IDEIA from $74 \%$ to $96 \%$. $^{6}$

Although the sensitivity of assays, based on examination of the serovars, varied enormously, when diluted clinical material was tested somewhat smaller differences were found between the final dilutions of samples that were positive by the most and least sensitive tests. Nevertheless, the order of sensitivity of the assays was similar to that found using the diluted serovars; the MT DFA and the MT EIA were again at the top of the range, although the rapid EIA (Clearview) performed better than before. Indeed, the MT DFA and Clearview differed only by a factor of $10^{2}$, whereas previously the difference had been $10^{4}$. This may reflect soluble LPS or free ribosomal RNA in clinical samples in which intact elementary bodies are present, but not in those in which they are absent, because no assay was positive when elementary bodies were not detected by DFA. These results may also reflect a problem experienced in preparing accurate dilutions of elementary bodies which, in cervical samples, have an affinity for host cells. Hence they tend to aggregate rather than being distributed throughout the sample. Aggregation would also occur with a chlamydia positive urine sample which might be considered a suitable alternative for comparative tests. Furthermore, caution should be exercised in the use of urines because not all assays have been validated for testing them.

It should come as no surprise that assays in which, in competent hands, a single elementary body may be detected, should be extremely sensitive. The lack of sensitivity of the other assays examined reflects their inherent inability to detect the LPS or ribosomal RNA extracted from small numbers of elementary bodies. This is a problem which may be compounded, in some cases, by only a small portion $(100-200 \mu \mathrm{l})$ of the original $1 \mathrm{ml}$ clinical sample being tested. This aspect should be addressed by the manufacturers. Perhaps it should be borne in mind that when culture was the only means of detecting $C$ trachomatis elementary bodies, the whole sample was tested and, indeed, passaged before a negative result was reported. The use of the whole sample in the Clearview assay may account in part for its better, although in our view inadequate, performance when clinical samples were tested.

The comparison of assays in large scale clinical trials may be valuable, but the results should be viewed with caution. It is possible to make any assay appear sensitive by comparing it with a suboptimal test, or to conceal a lack of specificity by excluding cultured specimens contaminated by those organisms which react non-specifically in the assay under investigation. In short, almost any result may ensue, albeit inadvertently. By using dilutions of laboratory grown $C$ trachomatis elementary bodies in a new assay and in one already in use, any laboratory will have a rapid, reproducible, and objective means of comparing their relative sensitivities. Furthermore, comparing the results of examining specimens by a new assay with those obtained on the same specimens with the MT DFA test or PCR will reveal the absolute sensitivity of the new method. Some will claim that such comparisons are unrealistic, but only when these are undertaken and the results are reported will the manufacturers of diagnostic tests improve their products to an acceptable level.

We thank Dr P E Hay and the nursing staff of the Jefferiss Wing of St Mary's Hospital, Paddington, London, for thei help in supplying the clinical samples, Mrs C B Gilroy for work involving the PCR, and the manufacturers of the various diagnostic kits for their cooperation.

1 Taylor-Robinson D, Thomas BJ, Osborn MF. Evaluation of enzyme immunoassay (Chlamydiazyme) for detecting Chlamydia trachomatis in genital tract specimens. $¥$ Clin Pathol 1987;40:194-9.

2 Thomas BJ, Evans RT, Hutchinson GR, Taylor-Robinson $D$. Early detection of chlamydial inclusions combining the use of cycloheximide-treated McCoy cells and immunofluorescence staining. F Clin Microbiol 1977;6: 285-92.

3 Palmer HM, Gilroy CB, Thomas BJ, Hay PE, Gilchrist C, Taylor-Robinson D. Detection of Chlamydia trachomatis by the polymerase chain reaction in swabs and urine from men with non-gonococcal urethritis. $f$ Clin Pathol $1991 ; 44: 321-5$.

4 Thomas BJ, Evans RT, Hawkins DA, Taylor-Robinson D. Sensitivity of detecting Chlamydia trachomatis eleD. Sensitivity of detecting Chlamydia trachomatis elementary bodies in smears by use of a fluorescein tional chlamydial isolation. $\mathcal{F}$ Clin Pathol 1984;37:812-6.

5 Thomas BJ, Osborn MF, Munday PE, Evans RT, Thomas BJ, Osborn MF, Munday PE, Evans RT, Taylor-Robinson D. A 2-year quantitative assessment of
Chlamydia trachomatis in a sexually transmitted disChlamydia trachomatis in a sexually transmitted dis-
eases clinic population by the MicroTrak direct smear eases clinic population by the MicroTrak direct smear
immunofluorescence test. Int $f$ STD AIDS 1990;1:264-7.

6 Thomas BJ, Osborn MF, Gilchrist C, Taylor-Robinson D. Improved sensitivity of an enzyme immunoassay IDEIA for detecting Chlamydia trachomatis. $\mathcal{f}$ Clin Pathol 1989;42:759-62. 